\title{
Mediated pedestrian mobility: walking and the map app
}

Eric Laurier, Institute of Geography, School of GeoSciences, University of Edinburgh

Edinburgh EH8 9XP

eric.laurier@ed.ac.uk

Barry Brown

Mobile Life Centre

SE-164 29 Stockholm, Sweden

barry@mobilelifecentre.org

Moira McGregor

Mobile Life Centre

SE-164 29 Stockholm, Sweden

moira@mobilelifecentre.org

Abstract

While walking has always been mediated, the arrival of smartphones with multiple apps has changed how we walk and how we use apps. In this paper we investigate the relationships of pedestrian-in-the-street and app-user-onscreen actions. We display and describe a series of intersubjective practices constituted by, and with, walking while using a mobile device. The video data used is from a larger study of pedestrians using smartphones in urban settings, with our analysis here turning on how a smartphone is used and interacted around to accomplish walking together. Our approach draws upon ethnomethodological conversation analysis (EMCA) studies of the sequential and category-based organization of mobile and on-screen actions. The analysis shows how walking actions (such as unilateral-stopping, turning, re-starting) are connected to map actions such as displaying the map, manipulating the scale, and monitoring the movement of the you-are-here dot. We conclude with remarks on the collaborative inter-subjective nature of walking with apps.

Keywords: walking, smartphones, map apps, wayfinding, action sequences, conversation analysis, ethnomethodology 
Introduction

Pedestrians don't just walk. They rush, they dawdle, they stroll, they amble, they circle, they pause, they stop, they edge past, they saunter, they plod, they advance, they retreat, they backtrack, they lead, they follow. Walking happens as a host of more prepositional, intentional and consequential actions: they walk toward, they walk away, they walk off, they walk into. Nor do pedestrians make naked contact with the places where they are walking: hiking shoes protect their feet while hillwalking, price tags and labels shape their trajectory while shopping, podcasts envelope them in comic dialogues while they walk to work.

In this article, we pursue and extend, then, the idea of that there is no such as thing as walking-initself. Our goal is not to produce 'the differential configurations of self and landscape' (Wylie 2005: 336) but rather to move toward the intersubjective production of mediated mobile practices. Our practices of walking in the city are not all the same, nor are they the same as walking barefoot in the park. They are mediated by the ancient technologies of shoes, pavements and pedestrian crossings. We walk around the supermarket with a shopping list (Cochoy 2008), stand at a bus-stop reading the timetable (Watson 2009), move around art galleries in relation to an exhibit (vom Lehn 2013) and stand for a while in the street pausing to listen to a busker (Smith forthcoming). How we move through places on foot has been further transformed by the arrival of various new technological systems and their related media. The smartphone looms large as a device that we walk with, one that introduces multiple kinds of media into pedestrian practices. One of the significant 'new-yetold' media that it has brought to walkers is the map. The map app departs from pedestrians' preexisting paper maps in several ways: map apps have a 'you-are-here' dot and provide suggested routes between points, map apps can be re-scaled and rotated to align with the compass orientation of the device and they integrate with online search engines. They allow their users to drop pins and other markers into the map, to show them where things are in relation to their current location.

Walking is seldom somnambulistic: to be moving your feet at all is also to be taken to have a reason for doing so. When the customer walks up to the counter they are taken to be about to pay for their goods. Walking, then, not only glosses over the myriad forms of pedestrian movement, it glosses over the intended actions that constitute pedestrian practices. Austin's (1962) 'How to do things with words' was a landmark lecture series in how it explored language not as a referential system for making true/false statements, but as something we do things with. Research on mobility has built upon Austin and others' work in considering the performative role of mobility yet has missed Austin's careful differentiation of what specific ordinary words do. For instance, in his discussions of truth statements he examines the varying effects if three words that are commonly used in those statements (e.g. 'entails', 'implies' and 'presupposes'). In this article we deepen Mobility Studies' engagement with Austin's work by considering how pedestrians do things with walking while never letting go of how walking is done with things.

In what follows, we will examine how the practice of walking is central to reading the map app and how the of reading of the map app is reflexively, if asymmetrically, tied to the walking. Through examining pedestrians walking together we will explore how walking plays its part in making recognisable to one another what is happening on a moment-by-moment basis, whether each and other knows where they are going and where each and other will go next. Specific features of walking actions such as pace, trajectory, halts, turns and so on provide resources for making sense of the map together, and showing the sense we are making of the map since pedestrian movement is itself inscribed into the map. GPS technology places a 'you-are-here' dot on the map, it scrolls the 
map and it is drawn upon to establish the scale. As Frith (2012) puts it, the map apps allow an inversion of the classic state cartography. Rather than the states predetermining how the world will appear on the map, the users of map apps, in interactions with their algorithms and interfaces, establish the centrepoints, scales and layers of their own maps, and they do this on a moment-bymoment basis.

\section{Doing things with walking}

While Ryave and Schenkein (1974) opened their classic study of "doing walking” with a brief mention of 'crawling, hopping, running, cartwheeling, jumping, skipping' $(1974,265)$ they settled on navigation and the reflexive production and recognition of pedestrians walking together as their main focus (Ryave \& Schenkein, 1974). We can, however, garner hints of the actions produced by walking in their remarks on how single persons carefully manage their movement on the pavement to avoid being seen as doing activities like 'following' or 'joining'. Their work was picked up almost two decades later by Lee and Watson's (1993) studies of pedestrians in street markets, boarding trains and navigating crowded stairways. Lee \& Watson described how walkers, by their general positioning in relation to one another and relevant features of the environment, produce 'flow files' and undertake pathfinding. Moreover they demonstrated how walking in public produces a moral order manifest in the accountability of pace and preferences for minimizing disruption to movement (Lee \& Watson 1993).

It is only recently that these early studies have been returned to, in part as a response to the rise of mobility studies and how mobility then features in embodied practices (see the collections (Haddington, Mondada, \& Nevile, 2013; McIlvenny, Broth, \& Haddington, 2014). Growing out of ethnomethodology and conversations analysis (EMCA) the contemporary studies have closely examined how talk, gestures, objects and environments are interwoven with courses of action. This turn toward mobility has been through undertaking studies of the vehicular, or, to put it another way, how people do things with, and through, moving as a vehicle. The vehicular units in question might be pedestrians entering a building (Weilenmann, Normark, \& Laurier, 2014), a couple travelling by car (Mondada, 2012), a small aircraft landing (Nevile, 2004) or a couple doing Lindy Hop (Broth \& Keevallik, 2014). Across these varied settings the studies have been concerned with, firstly, how people jointly coordinate and accomplish their mobility on a moment-by-moment basis and, secondly, how they do this as particular categories of social unit.

In terms of the moment-by-moment organisation of action, the classic ethnomethodological questions of 'why that now?' and 'what next?' have, in a number of these studies of movement, been supplemented with 'why that here?' and 'where next'? For the vehicular unit in motion, by their very movement through the environment, they have a constantly changing 'here' which then also provides changing access to events and to perspectives. Motion also shifts the proximity and distance of the features of the environment, for driving: side streets, exits, entrances (Haddington $\&$ Keisanen 2009) or, for supermarket shopping: aisles, freezer units, bakery counters and bargain baskets. Concomitantly by their ongoing movement, each unit has changes in their sequential geography of where they have just been and could possibly go next.

Sensitive to the situated nature of walking as action, EMCA studies have examined how the category of the unit itself produces distinctive ways of walking while also being reflexively reconfigured by those mobilities. In considering the social categories of the mobile unit we are returned to the premise of theories of walking and just as there is nothing that is walking-in-itself, walkers are never just walkers (Mondada 2012; Broth \& Mondada 2013; De Stefani \& Mondada 
2014). It is not just that walkers are themselves different categories of group (e.g. traffic wardens, office workers, museum visitors) - they undertake varied practices that then shift their categorisation, so that in the case of museum visitors they may shift from touring the exhibits, to browsing in the museum shop and then on to standing together in the museum restaurant queue. By doing so they shift from producing, being recognized and responded to as visitors, to shoppers and then to diners.

The recent EMCA studies have shifted the emphasis from the early studies interest in the ongoing maintenance of walking together to the various reconfigurations that happen when groups do things like 'moving off' or 'moving on' (Mondada 21012a; Broth \& Lundström, 2013; Lehn, 2013). The ongoing configuration of the relative positions of group members and their talk mutually elaborate one another so that, for example, in de Stefani's (2013) study of supermarket shopping, one member of a joint unit can announce that he is going to get the bread by himself, begin to depart and thereby temporarily break the pair into two solo shoppers. What de Stefani highlights is that this is joint mobile action and that rather than the one who has departed being able to thereby do what he intended or not, next actions are oriented toward and being responded to by the other party in parallel or subsequently. In de Stefani's case, when one shopper departs, the other shopper does not stand still, but instead 'walks beside' without a word uttered. An incipient departure for choosing bread alone is cancelled by a walking action that means the bread will be chosen together. Through studies of couples and friends shopping togetherIt is not that walking actions need to be infinitely varied, de Stefani shows how such simple mobile actions as 'stop' and 'go' are central to the mutual intelligibility of shopping as a pedestrian vehicle. In this article, we draw upon and extend these EMCA studies to consider how walking actions are used by wayfinding tourists in relation to mobile media.

\section{Mediated walking}

There is a long history of the mediation between the walker and the ground, most obviously through boots and shoes but also through tracks, roads and pavements (Ingold 2004; Michael 2000). For the walker in the city, not only is the very ground smoothed and shaped to allow them to look up from their footing (Ogborn 1998), they are surrounded by a multitude of urban signage and advertising, as sketched out by Latour (2003) in his study of Paris. In it, he documents a city bristling with street signs, optical devices, receipt slips, guidebooks and more; Latour's meditation on the mediation of Paris preceded the widespread adoption of the smartphone by urban pedestrians. The phone has brought to our lives in public spaces, new forms of encounter and new forms of co-presence (Licoppe, 2013). It has facilitated game-playing on the move and thus brought a changed way of playing together (Richardson, 2013), some games making 'remote' others co-present with the pedestrian (Southern, 2012). Map apps on smartphones arrive on the shoulders of a longer pedestrianisation and transformation of media, introduced through the use of new mobile devices. The transfer of telephony to the walker through the mobile phone brought its own changes to the assumed location of both the caller and the called (Ling, 2004; Weilenmann 2003). Mobile phones also brought SMS text messaging and consequently new practices of writing and reading as forms of being together with remote others (Harper et al 2006).

Walking through crowded urban spaces (or equally through empty rural spaces) has become hybridised with a series of practices previously found in other spaces: listening to music, gossiping with remote friends or ordering tonight's dinner (Frith, 2012). As the smartphone has enfolded further digital media (e.g. Google search, Wikipedia, Youtube, Yelp) it has brought ever more practices to pavements: searching, checking recommendations, playing games and, as we focus on in this article, following maps (de Souza e Silva \& Sutko, 2011; Brown et al 2015). The very walking 
of the vehicular unit is registered in the media by the system, a delegation of the work that a diligent map reader might have undertaken with pencils, compass, street signs and landmarks. Meantime the mobile device is not only tracked by GPS and made sense of by the system; the walkers take it out of their pocket, raise it close to their eyes, show it to or hide it from other walkers. As we noted above, the device itself has a mobility in relation to the talk, gestures and courses of action of the walkers. Our interest is in map practices now remediated by the map app, the movements of smart-phone itself and how map apps are then occasioned in particular ways within the pedestrian practices of wayfinding.

Wayfinding accomplished through mediated walking practices

As a walking unit, tourists move through the city in distinctive ways while also drawing upon their individual and relative mobility as a resource. For example, while one member of the group stops to consult the guidebook, other members can walk around inspecting the surrounding environment for street names, information signs, landmarks etc. (Laurier \& Brown 2008). By the member's pedestrian practices of 'walking away' from the immobile guidebook-reader the other members of the group categorise themselves as 'doing reconnaissance'. Having walked away to examine the surrounding environment, there are then expectations that they will return with information relevant to their wayfinding task.

It may not be initially clear the difference that walking as a particular mode and material arrangement of mobility makes to wayfinding with map apps. To turn briefly to GPS navigation systems in cars, while this is obviously a vehicular social unit moving together, the car passengers have fixed relative positions that are not easily interchangeable. The GPS units are usually also fixed to the dashboard as part of the ecology of the car interior. Once car travellers are settled into their seats they stay in them. From their relative positions, drivers and passengers can gesture with eyes and hands, turn their heads, and lean their torsos, but they are otherwise restricted in their mobility in relation to one another (Brown \& Laurier, 2012; Haddington \& Keisanen, 2009). Pedestrians using a map app on a smartphone are a different entity because they have distinctive embodied ways of walking together. They can individually 'stop' and 'go', change their relative pace thereby 'falling behind' or 'rushing ahead'; they can adjust the gap between their bodies, they can break apart their vehicle to let other pedestrians through or shift from side-by-side to single file to pass through narrow gaps or doors (Conein, Félix, \& Relieu, 2013). The device itself can be moved into and out of pockets and bags, closer and further away from eyes, tilted toward or away from the other walker and so on. With the new resources that map apps provide, they require different sorts of intersubjective mobile inquiry and are still settling into how we way find and way fare. We hope then in what follows there will be some novelty in examining how a particular map app is drawn upon and itself re-drawn during practices of walking as tourists in the city.

\section{Methodology}

The recent generations of lightweight video cameras developed for extreme sports have leant themselves to changed recording techniques for moving cameras with mobile subjects (see Brown et al. 2008; Spinney 2011). Following multiple camera techniques developed by McIlvenny (2013), for recording cyclists, we asked our project participants to each wear cameras that sat on their chests. While for McIlvenny's cycling studies, bicycle frames and riders' helmets provide several points for attaching cameras to then capture forwards, rearwards and sideways angles, we did not want to overburden our participants with cameras. Consequently each project participant had only one camera which then captured a chest height view ahead but also allowed us to work out the 
orientation of their torso, their direction and speed of walking. Depending on their orientation toward one another it produced recordings of each other body orientations and gestures from time to time. Other researchers (e.g. Licoppe \& Figeac, 2013) have used camera glasses that better capture head movements, glances, other gestures and inspection of smartphones at head height. However our preference was for the better quality images produced by sports cameras and they also allowed us to feed a lapel mic into them for better sound quality. The major drawback of our set-up is that we seldom see facial expressions and miss a number of gestures, objects and elements of the environment that will have been in the moving visual field of the participants. Not all of these absences are solved by camera glasses however because these cameras move with the head rather than the eyes of participants. During our data gathering we also installed screen capture apps on our participant's own phone or provided them with a project phone with the software already installed.

The recordings from the cameras and screen capture were transcribed as graphic transcripts (Laurier 2014). The format merges elements of the Jeffersonian conventions for transcribing conversations with those of graphic novels. Where speech bubbles overlap, participants' speech is overlapping. Equally where speech bubbles are placed far apart this indicates gaps between speaking. The temporal precision of textual transcripts is sacrificed in favour of, both the ease of reading of graphic transcripts and, more importantly, the visual details that they allow readers to register without lengthy textual descriptions.

We recruited 15 participants in the cities of Stockholm and London, 5 of these were individuals and 5 were couples or friends. Our set-up for gathering data was a hybrid of classic task-based wayfinding experiments and ethnographic studies of map use. The study participants were asked to go on a 'typical day out' which was convergent with what they were planning to do when they were recruited. We collected 24 hours of video data that was then edited into smaller fragments around wayfinding events and the use of map apps. The fragment we will draw upon in this article is a specimen, which is rich in material for analysing the interconnection of walking actions and the map app. It involves two American tourists, ('Carol' and 'Dani') realizing that they have made a mistake while walking toward a destination for lunch, and then repairing their mistake by consulting the map app.

\section{Walking as merely walking and checking the route}

As we have argued above, when people walk alone or together it involves much more than simply walking together side-by-side. Questions of where walkers are going next, who knows where the group is going next and whether they are more or less lost, are posed and answered through the combination of talk and walking actions. The production and recognition of one another's walking actions involves adjustments of pace, changes of direction, starting and stopping. In this first section we will examine the walking actions of the tourists where their pace and direction simply continues the journey itself.

The map app on Carol's smartphone has been used earlier to plan their route, identifying a short list of landmarks and street names. The underground stations along the main street that they are walking past were picked out as landmarks. In passing (or not passing) the landmarks that were earlier identified, the map is then made sense of in a reflexive relationship with the journey that they are making (Liberman 2013). Consulting the map is shaped by the very features of the urban environment that were picked out on each earlier consultation of the map app. 
By virtue of having her smartphone out and checking the map app at the outset of their journey, Carol has also become accountable for this planned route. Wayfinding in groups, be they formal like a ship's crew or informal like friends on a day out, involves distributing labour and accountability. Carol has acquired the responsibilities of being the map reader and being accountable for that as part of their journey in search of lunch. However, while these responsibilities remain potentially relevant during the journey, whether the walkers shift in and out of their map reading incumbency depends upon the other things they are doing amidst the walking activity. Walking in the city has a permeable quality because many other activities to seep into it.

In Transcript 1, Carol \& Dani are approaching the entrance to an underground railway station. The camera angle is forwards from Carol's mid-riff. The station entrance is on the street corner just beyond the red carpet entrance. Dani is walking on Carol's right hand side, out of shot.
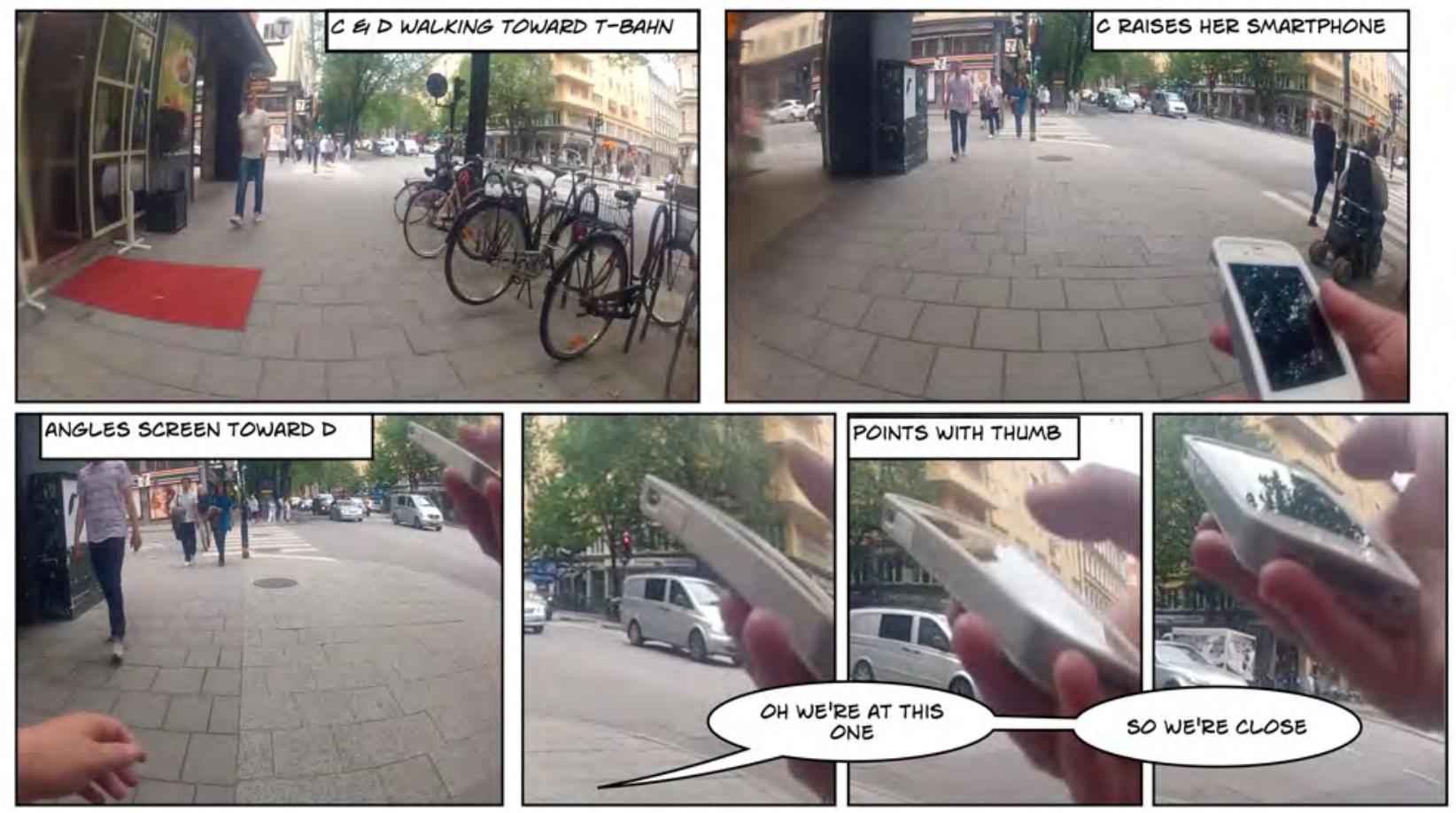

Transcript 1. Walking together, checking and showing smartphone

Closing in on the station entrance coincides with a lull in their conversation. Carol, by shifting the position of her smartphone, initiates a new course of action around the smartphone, which brings back her incumbency as map reader. However, for Dani (on the right), what is being initiated is not immediately apparent, given that there is an array of actions that may be initiated by raising a smartphone - such as answering an incoming call, checking a text message, taking a photo, checking email and, of course, checking the map. In continuing her gesture by then angling the smartphone toward Dani, Carol offers what is on screen for Dani. She then goes on to say, 'oh we're at this one', the oh-preface marking an epistemic change (e.g. it's a discovery that they are at this underground station). The referent of 'this one' being established by the timing in relation to: what they are currently walking past and her pointing over the screen at a station on the map (see Transcript 2). Dani has only minimal involvement, which is unsurprising considering her distance from the screen. Carol had to move the smartphone close to her eyes to inspect the map app closely enough to read road names etc. By contrast Dani merely glanced at the smartphone screen when it was tilted toward her in Transcript 1 , the distance combined with her glancing indicative of just such minimal involvement. 
Throughout this checking of the smartphone there is no change in the pace or direction of their walking and, in that sense, the journey continues onward without alteration or correction. What Carol's comment, 'so we're close', accomplishes is preparing the pair of them for the more detailed checking and approach-work that will be required on reaching their destination. The walking has shifted from walking past one landmark after another, to walking toward their journey's final destination. In sum, we have seen how the media is drawn upon smoothly to maintain continuous walking while wayfinding work is undertaken. Things however do not continue to be so smooth.

\section{Unilateral stopping}

For pedestrians walking together in the city, coming to a halt jointly without a verbal request is part and parcel of walking as a group. Pedestrians stop at the edges of roads when they cannot cross, they stop to take photographs, to let people pass or make their way through entrances and exits (Weilenmann et al. 2014). Closely coordinated stopping is possible because of the walkers' shared access to the many features of, and events in, the environment that call upon them to pause their journeys or stop travelling entirely. In fact, between Transcript 1 and Transcript 2 Carol and Dani have just passed through one of the most common urban features which make stopping relevant: the edge of the pavement where it meets the road.

Carol and Dani are advancing together continuously until Carol unilaterally stops (see Transcript 2a). Stopping walking can be done more or less abruptly and we have examples in our corpus of walking coming to a gradual stop. Here, when Carol stops, she stops sharply. Had she produced her stop more gradually then Dani might still have stopped in tandem with her. Moreover she is not at a point where stopping was already spatially relevant (e.g. the earlier edge of a pavement).
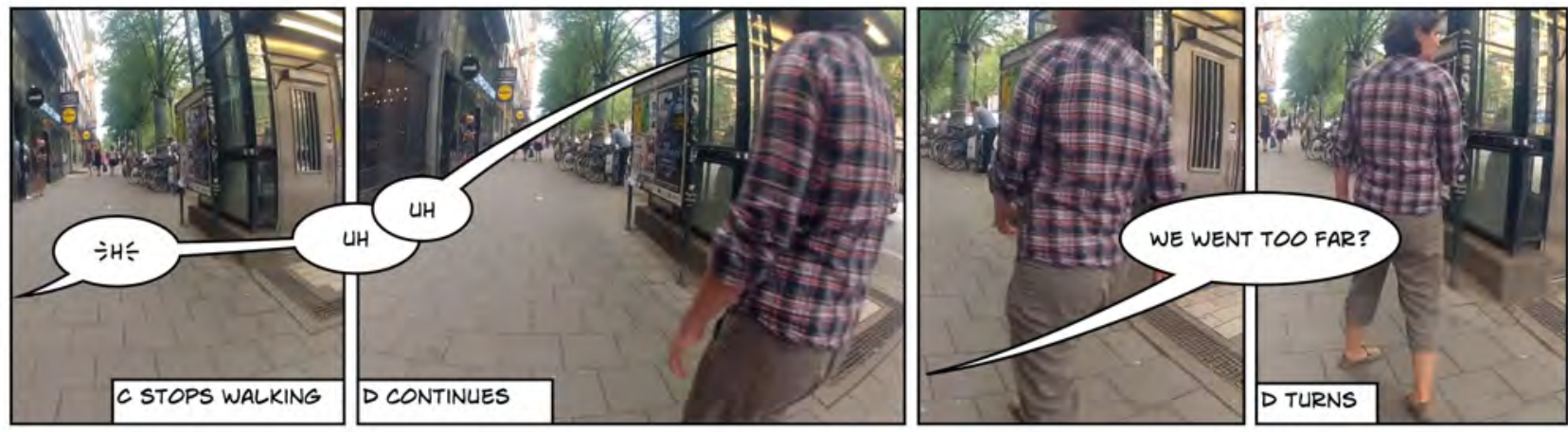

Transcript 2a: Unilateral and sharp stopping by $C$

However Carol's stopping is neither quite as abrupt nor unannounced as it might seem from Dani having missed it and walked on for several steps. Having checked her smartphone, Carol did not put it away and was still inspecting it as they crossed the road, having had to restart the maps app (we will look at that in more detail below). Moreover, the stop is pre-figured by an audible sigh. From the sigh, Carol then further prefigures difficulties by an 'uh' which Dani also produces in overlap (panels 2 and 3 of Transcript 2a). It may be that Dani's 'uh' precedes a question in response to the sigh over what Carol's difficulties are. In addition, Carol had mentioned that they were 'close' to their destination and it may be that Dani then anticipates problems around locating their destination given they have never been to it before so do not know what it looks like nor where it is.

Carol begins to account for her unilateral stop by saying 'we went too far?' (panels $3 \& 4$ ). The question is an account, which fits nicely with an immediate stop, which could itself precede turning back on their path as a likely next walking action. Carol's querulous tone may mark her own 
scepticism over what she has seen on the map app given that she had not detected any problems on passing the train station only three minutes earlier. Dani on hearing the question turns tightly around to re-orient herself both to a backward direction from their previous ongoing trajectory and to create a face-to-face spatial formation with Carol (see Mondada 2012a on changing formations). While unilateral stopping can accomplish various things, the responses to it are limited. The other walker(s) can also then stop or can continue walking. In de Stefani's (2013) work on supermarket shopping, when one shopper stops to inspect goods the other can continue ahead or also stop and join them in inspecting their goods. For the wayfinders, when the map reader stops, the other members of the party best not continue given that it is quite whether they should be walking ahead at all, that is in question.

\section{Walking as a you-are-here dot}

Map applications on smartphones ('map apps' such as Google Maps) have brought the you-are-here blue dot into common use. In the process, the previously fixed 'you-are-here' dot now moves across the map as its users move through the environment. Map apps depart from older paper maps in several other ways: they provide maps that can be re-scaled and rotated to align with the compass orientation of the device. We might expect that this combination of the locational resources of the you-are-here dot and the auto-rotation of the map would allow us to easily locate where we are while our journeys progress, given that they do the work of alignment of the map with the environment for us. Yet these very manipulation of media require further sense-making (Laurier \& Brown, 2008) and the things that we do with maps when we are on the move are much more than locating where we are (Brown \& Laurier, 2005).

Part of the reason that a unilateral stop occurs is that Dani does not have shared visual access to the map app. Before the unilateral stop, if we look at what was happening on Carol's screen, the map app had restarted, and Carol had re-typed the search term for the destination they had agreed upon earlier (panel 1 of Transcript 2b). The map re-appears on her map app when they are mounting the pavement. Carol was sighing, perhaps due to having to type their destination for a second time, when the map app restarted. The problem pre-figuring 'uh' occurs when $\mathrm{C}$ sees the red pin of their destination shift on the map app (see transcript $2 \mathrm{~b}$ inset boxes). It is this change of state on the map app that Carol responds to with a sudden unilateral stop.
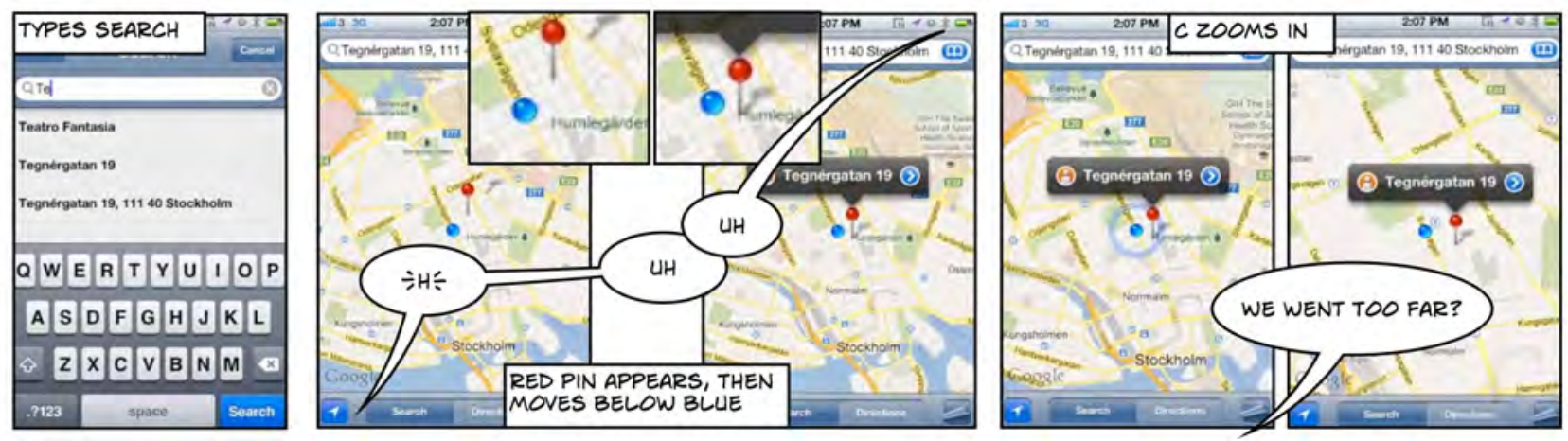

Transcript 2b: The you-are-here and destination dots

On each check of the map, Carol checked that their dot has moved further along their route, which is up the yellow street, prominent in the middle of the map. The trouble that Carol detects is that the red destination pin has shifted from being above the you-are-here blue dot, to below it. In response to the trouble $\mathrm{Carol}$ both lifts the device closer and pinches to zoom into the map for more detail. However, as we noted earlier in her comment, 'We went too far?', her tone remains sceptical 
or in some way uncertain over whether there really is a problem given her process of checking landmarks as they went along. The questioning tone is over the claim made by the map app even while, by being said aloud, it provides access to the mistake that Carol and Dani might have made in their journey.

While the longstanding convention for walking with paper maps has been that we work out landmarks and check them off as we pass them, the map app shows our location with a dot or similar icon. In the map app, the dot itself moves along the route that we have planned, or indeed off the route, depending on whether we deviate from the suggested route. Through its movement the you-are-here dot is aligned with the direction of our walking. A common trick we found pedestrians using was walking forward slightly to see where the blue dot moved on the map app, as a way of checking on their direction. Or, as is the case here, checking the movement of the dot as part of checking on the physical landmarks that they were passing. There is in the map app then a new set of resources for establishing where we are at any point in our journey.

\section{Reconnaissance and re-stopping}

To summarise our findings so far, we have looked at the smooth progression of walking with a map, the use of a unilateral stop by the map-reader that was, in part, generated by having asymmetric access to the smartphone and, further, we considered how the map app is used in making sense of where walkers are. We will now examine the distribution and articulation of wayfinding work in walking-actions as each walker moves in relation to another. Remembering that where they are and where they should go next is in question, we see how each party tries to help establish answers to these questions.

If we consider the earlier distribution of the wayfinding work, Dani had been journeying with Carol but only peripherally involved in the wayfinding (e.g. through being shown the device as they passed the train station entrance). After Carol's unilateral stop and account, Dani's involvement increases through an immediate mobile reconfiguration. In the first and second panels of transcript 3, Dani pivots rapidly around, continues walking while then also pointing toward the other side of the road. As part of this mobile reconfiguring, she jointly asks a wayfinding-relevant question of Carol as the map-reader. The question shows an understanding of Carol's immobility as being momentarily 'stuck'. Dani's pointing gesture is prolonged as she takes a few steps in the new direction that would allow them to backtrack and begin a search for the destination. In short, she is quickly attuned to the problem and seeking to establish the general direction of their destination.

We see the complex projectability of gesture and action that walkers can achieve in Dani's bringing together of two orientations: her direction of walk is in one direction while her pointing is in another. Her walking is toward some other walking-relevant target (e.g. the pedestrian crossing visible in the final frame of Transcript 3a) while her gesture is dealing with where she should look for their destination. What we can detect from Carol's camera movement (see the changing perspective in each panel in Transcript 3a) is that Carol rotates to converge with the direction of Dani's onward movement and by panel 4 appears to have faced toward the pedestrian crossing as well thereby bringing their projected next actions into alignment. 

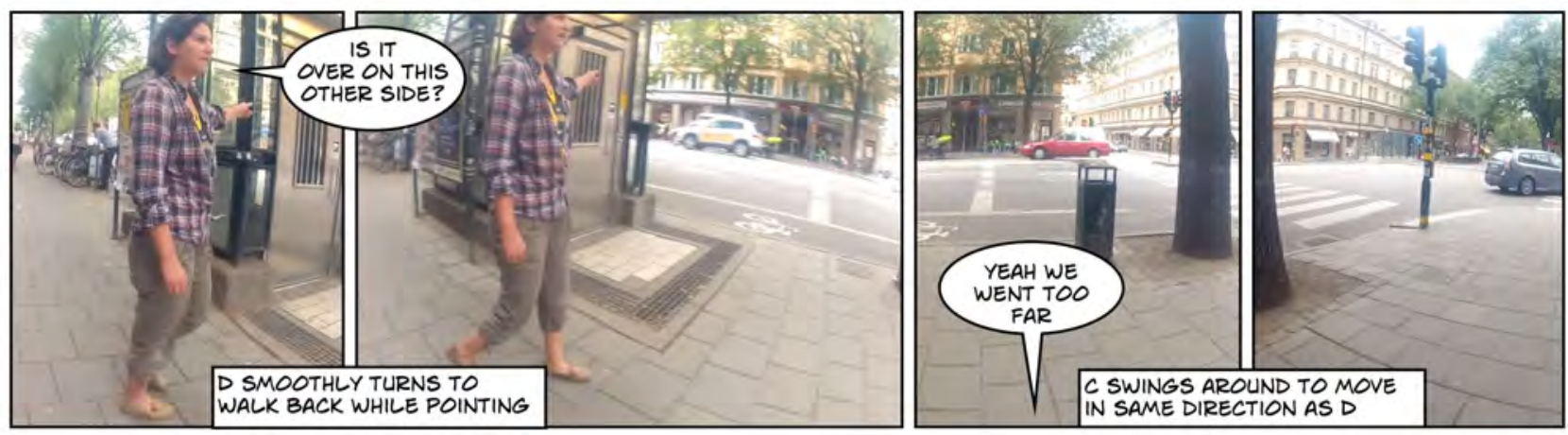

Transcript 3a: Dani's reconnaissance and Carol's confirmation of their mistake

Carol's response is more complex than moving together with Dani to the pedestrian crossing: even though she uses her walking actions to follow those of Dani, she ignores Dani's question 'Is it over on this other side?', instead answering her own earlier self-directed question, 'We went too far?', by providing a self-confirmation that, 'Yeah we went too far'. However it is not so much that she is ignoring Dani, because whether they have actually overshot is not something that Carol has established. On epistemologically thin ice, Carol is not moving too far toward cross the street until she has clarified whether there is a problem at all with their wayfinding or whether the problem is with the map app or with her current reading of the map app.

This parallel course of involvement continues when, after Carol begins to move she then re-stops. This second stop, after re-starting in the new direction, is accounted for with 'I think' as then being generated by her continuing uncertainty over whether they have overshot (which can be seen on the screen capture in Transcript 3b) and their movement. By zooming in on the map app, Carol has brought up street names and a more detailed view of the city blocks. Dragging the map up slightly (Transcript 4b) seems to help her become certain that they have gone too far rather than that there is some other problem.

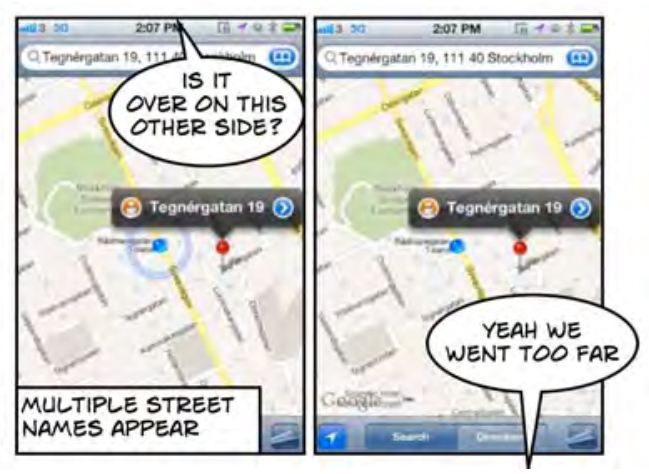

Transcript $3 b$.

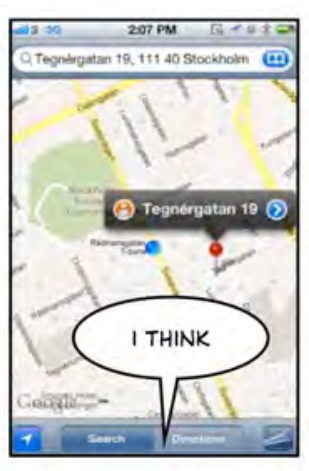

Transcript $4 b$

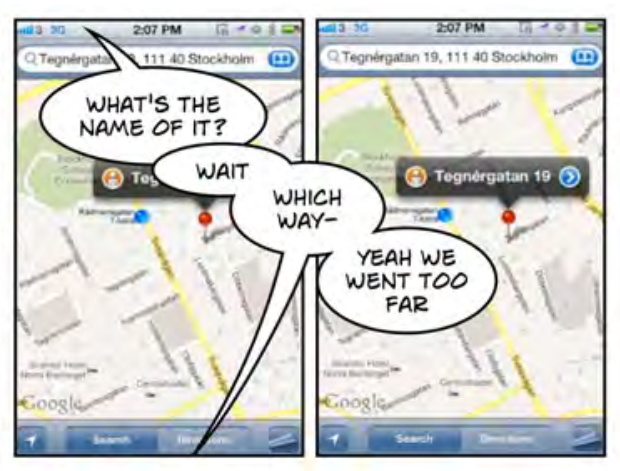

Reading the street names and adjusting the map

What the re-stop accomplishes, then, is putting their back-tracking on hold while Carol then examines the map app for a second time. Her re-stop is visible to Dani who then makes a few small steps to become proximate to Carol, showing by her walking an awareness of the need for further scrutiny of the map app. In this close formation Dani, does not simply wait on an answer from Carol reading the map app but makes a different request for a name of a street for Dani herself to look for. In her previous request she was asking for the area to look toward, now with Carol and Dani having an aligned orientation toward a tableau of shops, cafes and restaurants Dani asks for a name (see similar orientational work in the city street in Mondada 2009). With a name she could then scan the names of premises in the tableau. Carol holds off Dani's request with a brief, 'Wait' 
(panel 2, Transcript 4a). Carol then raises her own difficulties around their movement (e.g. 'which way') before finishing by becoming more certain again. She does this through repeating the phrase 'we went too far' for a third time so that it shifts from being a skeptical question to an affirmative conclusion.
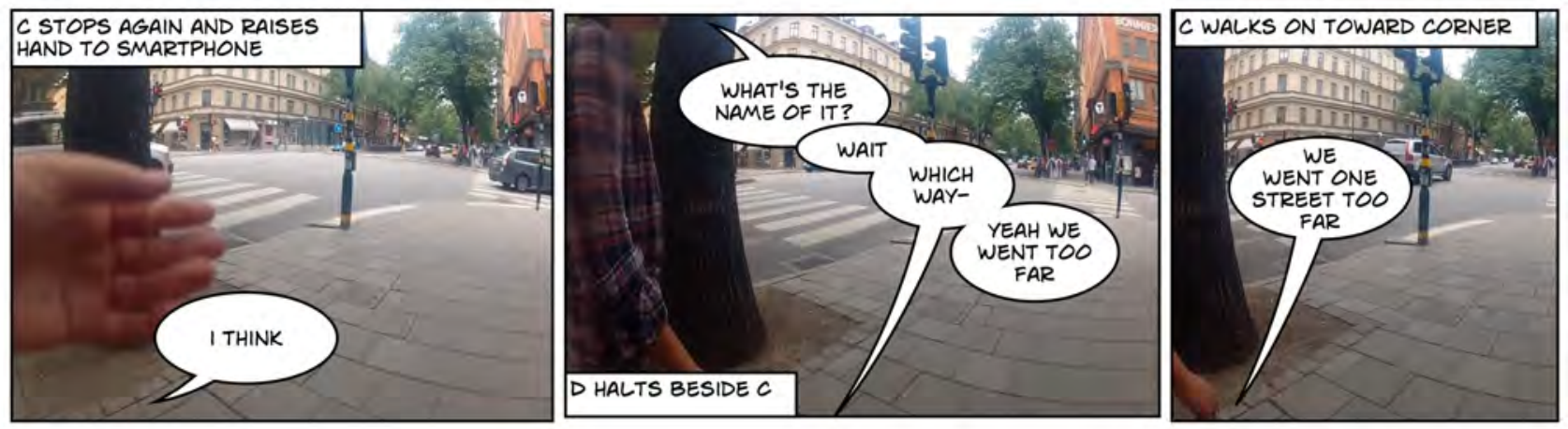

Transcript 4a: Carol re-stops and continues her map-reading

Aside from the re-stop putting their progress on hold, it also rearranges the pair's orientation toward one another and to the street features ahead. From the 'hold' position it is then Carol who re-starts their walking and thereby, Carol also shifts from becoming the uncertain map-reader to showing she knows where they are and where they are going. In terms of the relationship between walking and who knows what (Mondada, 2013): this brief pause in Carol's walking is used to mark her uncertainty about continuing with the adjustment to their route. Having made sense of the map app in relation to their journey, Carol then makes visible that certainty by 'setting off with a trajectory that re-initiates the move toward the crossing point.

What this complicated fragment of the interaction shows is how the reading of the media is reshaping their walking and being reshaped by their walking actions, not only on a moment-bymoment but in parallel courses of action one of which orients towards inspecting the urban environment and the other from inspecting the media (see also Haddington et al 2014). Moreover it underlines how a walking unit can distribute the wayfinding tasks amongst its members, even if, in this case, the party seeking to do some reconnaissance work is not given the information she would need to do so.

\section{Leading and following}

In the previous section we detailed how checking the map app and the city environment were accomplished through the sequences of walking stop/starts and questions: Dani, becoming the scout, turning around while pointing, Carol, becoming the map reader stopping, turning, then stopping again. We left Carol and Dani at a point where the map reader had become certain from her careful map reading that they have gone 'one street too far'. In this section we will examine how Carol makes it recognizable that she is now confident about where they are going. The walking action that Carol produces is to shift ahead of D's relative position. Rather than create a mobile configuration that is side-by-side, or single-file, she produces a step-ahead (see Transcript 5). The walking unit then becomes organised into one person leading and the other following a step behind. Carol's shift to leading the group is based upon her consultation of the map that she continues to check during her walk toward the pedestrian crossing, (see panel 2 of Transcript 5). 


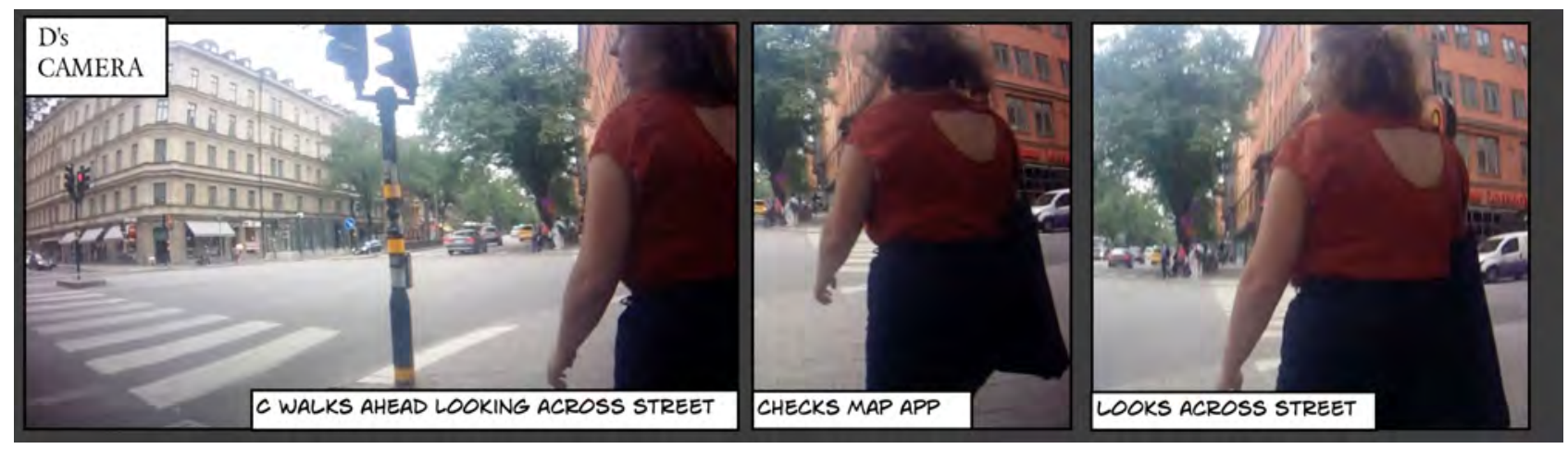

Transcript 5 (from Dani's perspective): Walking ahead of the other

It is hard not to feel that Dani has to some extent been sidelined in the wayfinding task given that she was not supplied with a name and thus was not able to search the neighboring environment for it, (an example of this can be found in Laurier and Brown 2008). However, Dani does not give up and although Carol is pressing ahead Dani then speeds up to close in on her again (see Transcript 7). While attempting to close the gap, Dani pursues the knowledge that will increase her parity in the wayfinding work by shifting from a request for a name to just an orientational question about their destination point on this street. Not only does Dani ask a further question in relation to finding their destination, she recreates a side-by-side arrangement of the walking pair so that as a walking unit, the leader-follower arrangement is being potentially ended. By her walking actions, in a reflexive relationship with her requests for information, she is managing her epistemic status in relation to their wayfinding as tourists. It is at this stage that she finally receives an answer to her 'side' question from Carol.

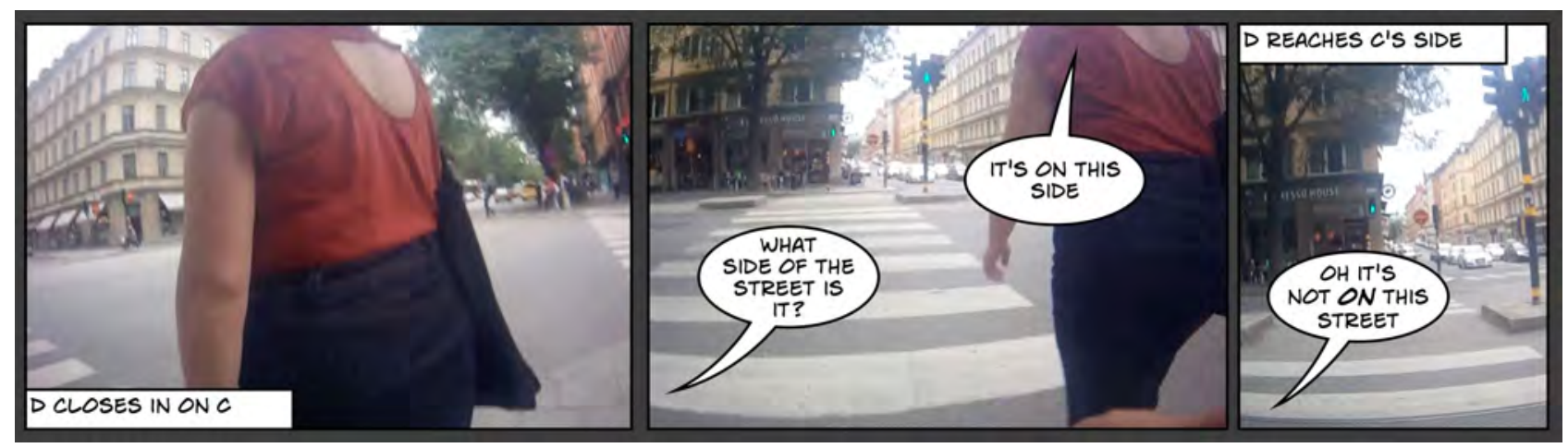

Transcript 6: Accelerating to recreate a side-by-side

Dani has an oh-prefaced realisation (see panel 3 of Transcript 6), before expressing her misapprehension that their destination was in the immediate area (e.g. 'not on this street'). Despite her desire to do so, Dani could not have helped solve their wayfinding problem by looking around for their destination because it was still beyond their visual access on the far side of a city block. What leads Dani to this realisation is hard to determine, but we can speculate that it is in the walking-action of Carol. Carol is walking ahead but she is not looking around at names of the places on the other side of the street and she does not point at anything. She only walks toward the other side of the street. In other words by the absence of the embodied qualities of inspecting from Carol's walking that would show that the destination is close enough to be looked for, Dani is able to establish that their destination is, 'not on this street'.

In this final section, we examined firstly how the leading and following configuration is assembled by Carol thereby re-establishing her as the map reader certain of where their destination lies. Secondly, we showed how Dani caught her up and re-established a side-by-side walking 
configuration, which is indeed the format for walking together as acquaintances or friends. Were Dani to have dropped behind Carol it would have been toward showing reluctance, dismay, tiredness or some other problem with their current togetherness as friends exploring a foreign city. Thirdly, we also touched upon how Carol's manner of walking at this point is made sense of in relation to their search for their destination. To be walking without scrutinizing the immediate environment was then analysed by Dani, also in relation to the absence of replies to her requests, as meaning that their destination was further than she had thought. In other words the journey itself is reflexively tied to expectations around walking-actions. When we near a new and unknown destination we adjust our walking to allow us to find that destination. 


\section{Conclusion}

If the first studies of the mediation and materiality of walking began, appropriately, where the foot hits the ground, in this article we have ascended to the mid-riff, shoulders and eye level. Ingold's careful consideration of walking showed us the, 'bias of the head over heels' (Ingold 2004, 331; see also Michaels 2000). In the rise of Modernity, feet were treated as farther and farther from thinking, even as they were augmented with shoes and disciplined through marching and other body techniques. For Michaels (2000) the boots are never simple intermediaries between the walker and nature, they also interfere, intervene and influence through pain, style, standardisation and erosion. It would be no surprise to say that the smartphone also interferes, intervenes and influences the walker. In the crowded streets of the city, the eyes that were freed from the ground by shoes and pavements to become Goffman's urban pedestrians that avoid bumping into others by 'scanning for other other people' (Ingold 2004, 238) - these eyes are now dragged downward again by the varied activities undertaken on smartphones. The smartphone-pedestrian does not move in the ways of the iPod-orchestrated-walker, that Bull $(2001,2005)$ described so well. By following two tourists for a few minutes while they are wayfinding in the city, what we have described is this evolving mediation of the smartphone and walking that picks up where Ingold and Michaels stop, at the feet of the pedestrian. We have brought thumbs onto map apps, walkers approaching pedestrian crossings, and the mutual and responsive orientation and trajectories of walkers.

Our article brings to their work on the mediation of walking not only this rapidly evolving technology that is the smartphone, it directs our attention to doing mobility together, and to responsive yet also divergent and convergent mobile actions. Where much of the work on walking has examined the link between the perceiving self and perceptible culture and environment (Wylie 2005), we have begun instead with how two persons are inter-subjectively producing their journey in the city. As such a central problem for people walking together remains around how walking is involved in producing the ongoing sense of: where we have been, where we are now and where we are going next. On pedestrian journeys there are jobs to do that are distributed amongst members of vehicular units, and these then also distribute locally determined accountabilities for onward (and backward) travel and the employment of media.

Through the analysis of video and screen recordings we have delineated how walking is one part of the gestalt of action which sustains the intelligibility (and unintelligibility) of what the walkers are doing in ongoing real time. We identified a number of walking actions: 'merely walking', unilateral stopping, turning while pointing (which is different from turning to point), re-stopping, re-starting, walking ahead, catching up, walking beside, changing direction. For each action, we described how walking actions were used within the emerging response to, and analysis of, the pedestrian tourists' problem of having overshot their destination, and in relation to one tourist's analysis and ongoing configuration of the map app. The reflexive relationship between reading the map app and the journey threaded through courses of action familiar from earlier studies of maps and wayfinding, but the map app brings with it new mediated practices, not least walking-the-dot. The latter may be analysed, as it was here, simply through accumulation of the previous checks of the you-are-here dot on the map or, as it was with other participants in our study, through actually walking forwards while checking to see what direction the blue dot then moved on the map.

In examining the complexities of how smartphones are implicated in pedestrian practices, what we hope is apparent, is that we have only just scratched the surface of the touchscreen's reconfiguration of, and by, mobility. If we consider for a moment, the ways in which smartphones are involved in 
friends being together and apart, catching buses, game-playing, looking up information, not to forget listening to music and a myriad other practices, there is much for mobility studies to understand about the mediation of not just walking actions but of course driving, cycling, travelling by bus, train and plane. We have attempted to move from the lone walker, to study a contemporary mode of walking: together with touchscreens.

Acknowledgements

A special debt it owed to Allison and James for their assistance in the revising and redrafting of this paper. To our study participants we also owe a huge thank you.

\section{References}

Austin, J. L. 1962. How to Do Things with Words: W. James Lectures Delivered at Harvard, 1955. London: Oxford University Press.

Broth, Mathias, and Fredrik Lundström. 2013. "A Walk on the Pier: Establishing Relevant Places in Mobile Instruction." In Interaction and Mobility, edited by Pentti Haddington, Lorenza Mondada, and Maurice Nevile, 91-121. Berlin: Walter de Gruyter.

Broth, Mathias, and L Keevallik. 2014. "Getting Ready to Move as a Couple: Accomplishing Mobile Formations in a Dance Class." Space and Culture 17 (2): 107-21. doi:10.1177/1206331213508483.

Broth, Mathias, and Lorenza Mondada. 2013. "Walking Away: the Embodied Achievement of Activity Closings in Mobile Interaction.” Journal of Pragmatics 47 (1): 41-58. doi:10.1016/j.pragma.2012.11.016.

Brown, Barry, and Eric Laurier. 2005. "Maps and Journeys: an Ethno-Methodological Investigation." Cartographica: the International Journal for Geographic Information and Geovisualization 40 (3). UT Press: 17-33.

Brown, Barry, and Eric Laurier. 2012. “The Normal Natural Troubles of Driving with GPS.” In. Brown, Barry, M McGregor, and D McMillan. 2015. "Searchable Objects: Search in Everyday Conversation.” doi:10.1145/2675133.2675206.

Brown, Katrina Myrvang, Rachel Dilley, and Keith Marshall. 2008. "Using a Head-Mounted Video Camera to Understand Social Worlds and Experiences." Sociological Research Online 13 (6). doi:10.5153/sro.1818.

Bull, Michael. 2001. "The World According to Sound Investigating the World of Walkman Users.” New Media E Society 3 (2). 179-97. doi:10.1177/14614440122226047.

Bull, Michael. 2005. "No Dead Air! the iPod and the Culture of Mobile Listening." Leisure Studies 24 (4): 343-55. doi:10.1080/0261436052000330447.

Cochoy, Frank. 2008. "Calculation, Qualculation, Calqulation: Shopping Cart Arithmetic, Equipped Cognition and the Clustered Consumer." Marketing Theory 8 (1): 15-44.

Conein, Bernard, Catherine Félix, and Marc Relieu. 2013. "The Visual Sense of Togetherness. Passing Through Doors.." Presented at MOBSIN 4, University of Nice, Sophia Antopolis, Copies available from the authors.

de Souza e Silva, Adriana, and Daniel M Sutko. 2011. "Theorizing Locative Technologies Through Philosophies of the Virtual." Communication Theory 21: 23-42. 
Frith, Jordan. 2012. "Splintered Space: Hybrid Spaces and Differential Mobility." Mobilities 7 (1): 131-49. doi:10.1080/17450101.2012.631815.

Garfinkel, Harold, and Anne Warfield Rawls. 2002. Ethnomethodology's Program. London: Rowman \& Littlefield Pub Incorporated.

Goodwin, Charles. 2003. "Pointing as Situated Practice." In Pointing: Where Language, Culture and Cognition Meet, edited by Sōtarō Kita, 2-32. New York: Lawrence Erlbaum Associates.

Goodwin, Charles. 2007. "Participation, Stance and Affect in the Organization of Activities." Discourse and Society 18 (1). Sage Publications: 53-73.

Haddington, Pentti, and Tiina Keisanen. 2009. "Location, Mobility and the Body as Resources in Selecting a Route.” Journal of Pragmatics 41 (10): 1938-61. doi:10.1016/j.pragma.2008.09.018.

Haddington, Pentti, Lorenza Mondada, and Maurice Nevile. 2013. "Being Mobile: Interaction on the Move." In Interaction and Mobility, edited by Pentti Haddington, Lorenza Mondada, and Maurice Nevile, 3-61. Berlin: Walter de Gruyter.

Haddington, Pentti, Tiina Keisanen, Lorenza Mondada, and Maurice Nevile. 2014. "Towards

Multiactivity as a Social and Interactional Phenomenon." In Multiactivity in Social Interaction:

Beyond Multitasking, 1-30. Amsterdam: John Benjamins Publishing Company.

Ingold, Tim. 2000. The Perception of the Environment. London: Routledge.

Ingold, Tim. 2004. "Culture on the Ground the World Perceived Through the Feet." Journal of Material Culture 9 (3): 314-40.

Kendon, Adam. 2004. Gesture. Cambridge: Cambridge University Press.

Laurier, Eric, and Barry Brown. 2008. "Rotating Maps and Readers: Praxiological Aspects of Alignment and Orientation." Transactions of the Institute of British Geographers 33 (2): 201-16.

Lee, John R. E., and Rod Watson. 1993. Interaction in Public Space: Final Report to the Plan Urbain. Paris: Plan Urbain.

Lehn, vom, Dirk. 2013. "Withdrawing From Exhibits: the Interactional Organisation of Museum Visits.” In Interaction and Mobility, edited by Pentti Haddington, Lorenza Mondada, and Maurice Nevile, 65-90. Berlin: Walter de Gruyter.

Liberman, Kenneth. 2013. More Studies in Ethnomethodology. New York: State University of New York Press.

Licoppe, Christian. 2013. "Merging Mobile Communication Studies and Urban Research: Mobile Locative Media, 'Onscreen Encounters' and the Reshaping of the Interaction Order in Public Places.” Mobile Media E Communication 1 (1): 122-28. doi:10.1177/2050157912464488.

Licoppe, Christian, and Julien Figeac. 2013. "Patterns of Gaze Switching in the "NaturallyOccurring' Uses of Smartphones in Urban Mobile Settings.” TELECOM ParisTech. Paris.

Ling, Richard Seyler. 2004. The Mobile Connection. San Francisco (CA): Morgan Kaufmann Pub.

McIlvenny, Paul. 2015. "The Joy of Biking Together: Sharing Everyday Experiences of Vélomobility." Mobilities 10 (1): 55-82. doi:10.1080/17450101.2013.844950.

McIlvenny, Paul, Mathias Broth, and Pentti Haddington. 2014. "Moving Together: Mobile Formations in Interaction.” Space and Culture 17 (2): 104-6. doi:10.1177/1206331213508679.

Michael, Mike. 2000. "These Boots Are Made for Walking...: Mundane Technology, the Body and Human-Environment Relations." Body and Society 6 (3-4): 107-26. doi:10.1177/1357034X00006003006.

Mondada, Lorenza. 2012a. "Garden Lessons: Embodied Action and Joint Attention in Extended Sequences." In Interaction and Everyday Life: Phenomenological and Ethnomethodological Essays in Honor of George Psathas, edited by Hisashi Nasu and Frances Chaput Waksler, 279-96. Plymouth: Lexington Books.

Mondada, Lorenza. 2012b. "Talking and Driving: Multiactivity in the Car." Semiotica 191 (1/4): 223-56. doi:10.1515/sem-2012-0062.

Mondada, Lorenza. 2013. "Displaying, Contesting and Negotiating Epistemic Authority in Social Interaction: Descriptions and Questions in Guided Visits.” Discourse Studies 15 (5): 597-626. 
doi:10.1177/1461445613501577.

Moores, Shaun. 2014. "Digital Orientations: 'Ways of the Hand' and Practical Knowing in Media Uses and Other Manual Activities.” Mobile Media E Communication 2 (2): 196-208. doi:10.1177/2050157914521091.

Nevile, Maurice. 2004. Beyond the Black Box. Aldershot: Ashgate Pub Ltd.

Ogborn, Miles. 1998. Spaces of Modernity. New York: Guilford Press.

Richardson, Ingrid. 2013. "Touching the Screen: a Phenomenology of Mobile Gaming and the iPhone.” In Studying Mobile Media, 133-51. London: Routledge.

Ryave, Alan Lincoln, and James N Schenkein. 1974. "Notes on the Art of Walking." In Ethnomethodology; Selected Readings, 265-74. Harmondsworth: Penguin.

Schütz, A. 1967. The Phenomenology of the Social World. London: Northwestern University Press.

Smith, Tim. 2012. "Street Performing Spectacles and Ordinary Life."

Southern, Jen. 2012. "Comobility: How Proximity and Distance Travel Together in Locative Media." Canadian Journal of Communication 37 (1): 75-91.

Spinney, Justin. 2011. "A Chance to Catch a Breath: Using Mobile Video Ethnography in Cycling Research.” Mobilities 6 (2): 161-82. doi:10.1080/17450101.2011.552771.

Stefani, Elwys De. 2013. "The Collaborative Organisation of Next Actions in a Semiotically Rich Environment: Shopping as a Couple." In Interaction and Mobility, edited by Pentti Haddington, Lorenza Mondada, and Maurice Nevile, 123-51. Berlin: Walter de Gruyter.

Stefani, Elwys De, and Lorenza Mondada. 2014. "Reorganizing Mobile Formations: When 'Guided' Participants Initiate Reorientations in Guided Tours.” Space and Culture 17 (2): 15775. doi:10.1177/1206331213508504.

Suchman, Lucille Alice. 2007. Human-Machine Reconfigurations. Cambridge: Cambridge University Press.

Watson, Rod. 2009. Analysing Practical and Professional Texts. Farnham: Ashgate.

Weilenmann, Alexandra. 2003. “'I Can't Talk Now, I'm in a Fitting Room”: Formulating Availability and Location in Mobile-Phone Conversations." Environment and Planning A 35: 1589-1605.

Weilenmann, Alexandra, Daniel Normark, and Eric Laurier. 2014. "Managing Walking Together: the Challenge of Revolving Doors." Space and Culture 17 (2): 122-36. doi:10.1177/1206331213508674.

Wylie, John. 2005. “A Single Day's Walking: Narrating Self and Landscape on the South West Coast Path." Transactions of the Institute of British Geographers 30 (2): 234-47. 